\section{Acetylating for avidity}

N-terminal acetylation of eukaryotic proteins is an important and widespread modification, but its impact on protein-protein interactions is poorly understood. The E2 ubiquitinconjugating enzyme Ubc12 cooperates with the RING E3 ubiquitin ligase Rbx1 and

a co-E3, Dcn1, to mediate transfer of the ubiquitin-like protein Nedd8 to substrate cullins. Dcn1 interacts with an N-terminal extension on Ubc12, and Schulman and colleagues now show that NatC-catalyzed N-terminal acetylation of the extension enhances the interaction between Dcn 1 and Ubc12, promoting neddylation of substrates. The crystal structure of an N-terminally acetylated Ubc12 peptide bound to Dcn 1 shows the acetyl group making direct interactions with residues lining a hydrophobic pocket in Dcn1. Additionally, the acetyl group enhances the Ubc12-Dcn1 interaction by masking the positive charge at the end of the N-terminal Ubc12 $\alpha$-helix; mutations in Dcn1 that can accommodate Ubc12's positive charge suppress the need for acetylation in vitro and in vivo. A stapled-helix Ubc12 peptide strengthens the Dcn1-Ubc12 interaction, indicating that conformational flexibility of $\mathrm{Ubc1} 2$ also affects the affinity between the two proteins. Exploring the effects of N-terminal acetylation on other pathways will give further insights into the mechanisms by which this modification regulates diverse cellular processes. The authors propose that disrupting N-terminal acetylation-mediated interactions may have therapeutic potential in many systems. (Science doi:10.1126/ science.1209307, published online 22 September 2011)

SM

\section{Regulated unloading}

The small G proteins Rheb and Ras, important in cell growth and proliferation, are farnesylated in their C-terminal domain. This posttranslational modification is important for membrane localization and adds a layer of regulation. The proteins are trafficked to different membrane compartments by their guanine-nucleotide dissociation inhibitor (GDI) PDE $\delta$, which sequesters the hydrophobic modification and solubilizes the G proteins. GDI displacement factors (GDFs) then unload the G proteins at their target membranes, but the mechanism by which this occurs is unknown. Wittinghofer and colleagues have examined the interaction between Rheb and PDE $\delta$ and how it is regulated by GDFs Arl 2 and Arl3, known binding partners of PDE $\delta$. They determined the crystal structure of PDE $\delta$ in complex with farnesylated Rheb (F-Rheb), revealing the hydrophobic pocket used by PDE $\delta$ to bury the lipid group. Most of the contacts between the two proteins occur at the farnesylation site, far away from the switch region of Rheb, explaining previous observations about the nucleotide-independent binding of G proteins to PDE $\delta$. A previous structure of Arl2-GTP-PDE $\delta$ showed the newly identified hydrophobic pocket of $\mathrm{PDE} \delta$ in a closed conformation, suggesting that Arl2 may regulate PDE $\delta$ association with F-Rheb allosterically. Functional data confirm that Arl2 can promote GTP-dependent dissociation of PDE $\delta$. Formation of the ternary complex Arl2-GTP-PDE $\delta$-F-Rheb accelerates the release of the farnesylated protein, and the authors identified residues on PDE $\delta$ involved in this allosteric regulation. These findings provide a model for how other GDFs may act to regulate their GDIs and point to another target site for development of drugs for the related oncogenic G protein Ras. (Nat. Chem. Biol. doi:10.1038/nchembio.686, published online 16 October 2011)

$M M$

Written by Inês Chen, Arianne Heinrichs, Steve Mason \&

Michelle Montoya

\section{Invading the nucleus}

Like many other viruses, adenovirus undergoes replication inside the host cell nucleus. The viral genome is packaged within a capsid that protects it from degradation and from detection by cellular defense systems in the cytoplasm, but the capsid needs to be disassembled before replication can occur. The viral capsid is far too large to pass through the nuclear pore, and how exactly the viral genome gets uncoated from the capsid and enters the nucleus is not clear. Now Greber and colleagues report that adenoviruses use a cellular motor, kinesin-1, to facilitate both processes. Using fluorescence and transmission electron microscopy analyses of adenovirus-infected cells, the authors observed that the disassembled viral capsids are associated with nucleoporins at the cytoplasm periphery. Because kinesin-1 is involved in anterograde transport of cellular and viral cargos, the authors tested kinesin-1 components and found that the light chains Klc1 and Klc2 and heavy chain Kif5C associate with adenoviral capsids and are required for their disassembly at the nuclear envelope and hence for viral infection. Kif5C was previously known to interact with the nuclear pore, resulting in activation of the ATP turnover by $\mathrm{Kif5} \mathrm{C}$ in the presence of microtubules. Although the interaction of Kif5C with the nuclear pore complex was required for viral infection, it was not necessary for capsid association with the nuclear envelope. The specific interactions of viral capsid proteins with kinesin-1 and with nuclear pore components were mapped. The action of kinesin-1 on viral capsids docked to the nuclear pore resulted in disruption of the nuclear pore complex. The authors propose a model whereby kinesin-1 motor activity pulls apart the viral capsid; this separates the capsid from the genome and disrupts the nuclear pore complex, thereby facilitating entry of the adenoviral DNA into the nucleus. (Cell Host Microbe 10, 210-223, 2011)

IC

\section{Mediator action}

It is largely unknown how specific histone modifications and the effector proteins they recruit are coordinated with assembly of the preinitiation complex (PIC) at gene promoters to achieve transcriptional activation. The chromatin remodeling protein CHD1, which is necessary for embryonic stem cell pluripotency, binds to nucleosomes that are trimethylated at histone $\mathrm{H} 3$ Lys4 (H3K4me3). To study the mechanism by which H3K4me3 contributes to active transcription, Carey and colleagues reconstituted $\mathrm{H} 3 \mathrm{~K} 4 \mathrm{me} 3$ - and $\mathrm{CHD1}$-stimulated gene activation on chromatin templates. Proteomics analysis of PICs assembled on unmodified chromatin versus $\mathrm{H} 3 \mathrm{~K} 4 \mathrm{me} 3$ chromatin showed the transcriptional activator-dependent recruitment of CHD1 to PICs on unmodified chromatin templates, and CHD1 recruitment to PICs was further enhanced by H3K4 trimethylation. Proteomics analysis also confirmed the activatordependent recruitment of the mediator coactivator complex. Mediator, which controls PIC assembly, is in turn needed for CHD1 recruitment. CHD1 stimulated in vitro transcription in a Mediator-dependent manner, and previously reported genomewide chromatin immunoprecipitation data revealed overlapping CHD1-binding- and Mediator-binding sites that correlate with active gene transcription in mouse embryonic stem cells. Together, these findings suggest that Mediator coordinates assembly of the PIC and recruitment of CHDI and that the PIC and H3K4me3 together provide specificity in targeting CHD1 to active genes. (Genes Dev. doi:10.1101/gad.17554711, published online 6 October 2011) 